\title{
Special section on testing and repair for software engineering technologies and applications
}

\author{
Ian Bayley ${ }^{1} \cdot$ Yan Cai $^{2} \cdot$ Patricia Machado ${ }^{3}$ \\ Published online: 1 February 2020 \\ (C) Springer Science+Business Media, LLC, part of Springer Nature 2020
}

The challenges of developing correct software are well known. Effective techniques are required both for software testing and repair. Manual program repair is time consuming and therefore expensive, and this fuels interest in automated program repair. The potential synergies between software testing and program repair are of particular interest, and they prompted this special section. A program repair may explain the reason for a failure and assist the debugging process, but likewise, a well-designed test case can provide the information needed for fault localization.

This special section was preceded by the conference SETA (Software Engineering Technologies and Applications), held in Tokyo as part of the COMPSAC (the IEEE Computer Society Signature Conference on Computers, Software and Applications). The issue received seven submissions, and after rigorous reviewing, only two were accepted, both focusing on dynamic testing and encompassing the test case generation challenges for concrete execution and symbolic execution.

The first article, by Esmaeel Nikravan and Saeed Parsa, is "Improving Dynamic Domain Reduction Test Data Generation Method by Euler/Venn Reasoning System." It proposes a dynamic testing approach to improve domain reduction-based test data generation by mapping each variable domain to an Euler/Venn diagram and utilizes the Euler/Venn reasoning system to select more accurate subdomains. This improves efficiency and accuracy.

The second article, by Maryam Abdul Ghafoor, Muhammad Suleman Mahmood, and Junaid Haroon Siddiqui, is "Extending Symbolic Execution for Automated Testing of Stored Procedures." This also focuses on the dynamic aspect of testing, namely, on dynamic symbolic

Ian Bayley

ibayley@brookes.ac.uk

Yan Cai

ycai.mail@gmail.com

Patricia Machado

patricia@computacao.ufcg.edu.br

1 Oxford Brookes University, Wheatley Campus, Oxford OX33 1HX, UK

2 Chinese Academy of Sciences, Institute of Software, Beijing 100190, China

3 Federal University of Campina Grande, Campina Grande, Brazil 
execution for test case generation and their corresponding database states for stored procedures. This improves effectiveness on generating test cases for finding more schema constraint violation bugs and user-defined exceptions.

As the guest editors, we thank the authors for their hard work in preparing their manuscripts and the reviewers for their detailed reviews. Finally, we thank Rachel Harrison, the Editor-inChief, and the editorial staff for their diligence.

Ian Bayley

Yan Cai

Patricia Machado

January 9, 2020 